\title{
USA Winter 2021 CoVID-19 Resurgence Post-Christmas Update
}

\author{
Genghmun Eng \\ PhD Physics 1978, University of Illinois at Urbana-Champaign
}

February 4, 2022

\begin{abstract}
We have successfully modeled every USA CoVID-19 wave using: $N(t) \approx \max \left[N_{o} \exp \left\{+\left(t /\left[t_{R}\left(1+\alpha_{S} t\right)\right]\right) \exp \left(-\delta_{o} t\right)\right\}\right]$,

where $N(t)$ is the total number of new CoVID-19 cases above a prior baseline, and $t_{R}$ sets the doubling time $t_{d b l}=t_{R}(\ln 2)$. The new parameters $\left\{\alpha_{S} ; \delta_{o}\right\}$ measure mitigation efforts among the uninfected population, with $\left\{\alpha_{S}>0\right\}$ being associated with Social Distancing and vaccinations; while $\left\{\delta_{o}>0\right\}$ is associated with mask-wearing, which gives a faster $\frac{d}{d t} N(t)$ post-peak drop-off. The predicted pandemic wave end is when $N(t)$ no longer increases.

Using data from $11 / 15 / 21-12 / 30 / 21$, our prior medrxiv.org preprint* showed this initial Omicron CoVID-19 wave had values that matched the initial stage of the prior USA Winter 2020 resurgence $\left\{t_{R} \approx 8.05\right.$ days; $\left.\alpha_{S} \approx 0.011 / d a y\right\}$, when practically no one was vaccinated. In addition, this initial Winter 2021 wave showed virtually no mask-wearing $\left\{\delta_{o} \approx\right.$ $0.001 \times 10^{-3} /$ day $\}$, making it capable of infecting virtually everyone. These parameter values indicated that the Omicron variant was likely evading the vaccines in people who thought they were protected.

As a result, stopping the Omicron CoVID-19 spread must once again rely on enhanced Social Distancing and mask-wearing, just like the initial pandemic wave in March 2020. Analyzing the USA follow-on data from $12 / 25 / 21-1 / 31 / 22$ shows that people did exactly that after the Christmas Holiday season, resulting in the following model parameters and values:

$$
\begin{aligned}
& N_{o}[12 / 25 / 21]=3,121,000 ; \\
& N[t=1 / 31 / 22] \approx 26,870,400 ; N[t=3 / 21 / 22] \approx 50,522,800 ; \\
& t_{R}=7.636 \text { days } ; t_{d b l}=5.293 \text { days } ; \\
& \alpha_{S}=0.03168 / \text { day } ; \\
& \delta_{o}=0.96 \times 10^{-3} / \text { day } ;
\end{aligned}
$$$$
\alpha_{S}=0.03168 / \text { day }
$$

for this wave by itself, with all prior waves subtracted out as a baseline. Combining all the USA CoVID-19 waves gives these updated totals:

$N_{\text {tot }}(t=[1 / 31 / 22]) \approx 77,122,000$ vs $N_{\text {data }}=75,399,650 ;$

$N_{\text {tot }}(t=[3 / 21 / 22]) \approx 101,476,500 ; N_{\text {tot }}(t=[6 / 10 / 22]) \approx 111,531,800$ assuming no future CoVID-19 Resurgence (with 4 Figures).

*(10.1101_2021.10.15.21265078)
\end{abstract}




\section{Introduction}

Each USA CoVID-19 wave ${ }^{\mathbf{1 - 7}}$, from the pandemic start (3/21/20) to the present day $(1 / 31 / 22)$, has been successfully modeled using this basic $N(t)$ function for the total number of new CoVID-19 cases above a prior baseline:

$$
N(t) \approx \max \left[N_{o} \exp \left\{+\left(t /\left[t_{R}\left(1+\alpha_{S} t\right)\right]\right) \exp \left(-\delta_{o} t\right)\right\}\right],
$$

with $t_{R}$ setting the pandemic doubling time $t_{d b l}=t_{R}(\ln 2)$, as in standard SEIR (Susceptible, Exposed, Infected, Recovered or Removed) epidemiology models. The new Eq. [1.1] parameters model mitigation efforts among the uninfected population, with $\left\{\alpha_{S}>0\right\}$ associated with Social Distancing and vaccinations; and $\left\{\delta_{o}>0\right\}$ associated with mask-wearing, which gives a faster $\frac{d}{d t} N(t)$ post-peak drop-off. The predicted pandemic wave ends when $N(t)$ no longer increases.

Given a total population of $N_{A L L}$, the uninfected population $U(t)$ is:

$$
U(t)=\left[N_{A L L}-N(t)\right] \text {. }
$$

Using Eq. [1.1] assumes $N(t)<<N_{A L L}$, so that pandemic saturation effects can be ignored. Also, SEIR models do not generally include what the $U(t)$ uninfected population is doing in response to the pandemic. In contrast, Eq. [1.1] was developed as a non-local extension of SEIR models, to account for how the uninfected population, as a whole, is mitigating the pandemic spread.

Each new USA CoVID-19 wave starts with a sharp rise in the total number $N(t)$ of new cases, while the $t=0$ point is chosen to be when the resurgence is first easily identified, with $N(t=0)=N_{o}$ being the number of cases above baseline at that time. Since Eq. [1.1] is empirically based, it does not predict when each new CoVID-19 wave will start, or what biological and social circumstances are causing the new wave. But once the CoVID-19 wave becomes established, Eq. [1.1] appears to successfully predict its time evolution.

Since the same few parameters in Eq. [1.1] have successfully modeled the time evolution of each USA CoVID-19 wave ${ }^{1-7}$, this result shows that the response of the $U(t)$ uninfected population has been similar for each wave, even if different dominating factors were driving the CoVID-19 resurgences.

All CoVID-19 data used here came from the open-source bing.com CoVIDTracker $^{8}$ database. The initial stage of the USA Winter 2021 CoVID-19 Resurgence started when the Omicron CoVID-19 variant became ascendant. Our prior medrxiv.org preprint ${ }^{7}$ covering data from 11/15/21-12/30/21 showed that this stage was characterized by:

$$
\begin{aligned}
& N_{o}(11 / 15 / 21)=107,000 ; \\
& t_{R}(11 / 15 / 21-12 / 30 / 21) \approx 8.05 \text { days } ; \\
& \alpha_{S}(11 / 15 / 21-12 / 30 / 21) \approx 0.011 / \text { days } .
\end{aligned}
$$

These values are similar to the USA Winter 2020 CoVID-19 Resurgence, which only had a fully-vaccinated rate of $\sim 0.3 \%$. Since the USA now has a significantly vaccinated population, it makes it likely that the Omicron CoVID19 variant is evading the vaccines.

While the prior 2020 Winter Resurgence had a significant amount of maskwearing $\left[\delta_{o}(2020) \approx 1.748 \times 10^{-3} /\right.$ day], the initial part of the 2021 Winter Resurgence was associated with virtually no mask-wearing: 
indicating that the Omicron variant was infecting people who thought they were protected. While Omicron vaccine evasion is now generally accepted, vaccination still offers protection against hospitalization from an Omicron CoVID19 infection. However, with virtually no mask-wearing, the Eqs. [1.3a]-[1.3c] values showed that this initial stage of the Winter 2021 CoVID-19 wave showed that it could infect virtually all susceptible people ${ }^{7}$.

This result similar to the initial stage of the USA Summer 2021 wave $^{5}$. That commonality shows that resurgences may be driven by people letting their guard down with respect to Social Distancing and mask-wearing. As this initial Winter 2021 CoVID-19 Resurgence occurred between Thanksgiving and Christmas, festivities likely contributed to this CoVID-19 surge.

However, right after the Christmas Holiday, when the number of new cases reached $\sim 3,120,000$ cases above baseline, the uninfected population began practicing enhanced Social Distancing and mask-wearing. This change is similar to what occurred during the latter portion of the USA Summer 2021 CoVID-19 wave $^{5}$, and it is modeled here in this update.

\section{Post-Christmas USA Winter 2021 Resurgence}

Using the USA Summer 2021 CoVID-19 Resurgence as a baseline, it showed that the USA Winter 2021 CoVID-19 Resurgence started around 11/15/21. The dots in Figure 1 show the total number of new CoVID-19 cases above the Summer 2021 USA CoVID-19 baseline ${ }^{\mathbf{5}-\mathbf{6}}$. The Figure 1 continuous line shows the original CoVID-19 projections for this initial stage of the USA Winter 2021 CoVID-19 Resurgence ${ }^{7}$, using data from 11/15/21-12/30/21.

Figure 2 includes follow-on data after this initial USA Winter 2021 CoVID19 Resurgence, with data and models updates for 12/25/21-1/31/22, covering the post-Christmas CoVID-19 surge. Almost immediately after the Christmas Holiday, the uninfected population returned to enhanced Social Distancing and mask-wearing, creating a distinct change in the $N(t)$ function after 12/25/21.

Modeling the 12/25/21-1/31/22 period of the USA Winter 2021 wave in Figure 2 shows that this CoVID-19 wave can subside prior to infection of the entire USA population. This latter portion has these model parameters:

$$
\begin{aligned}
& N_{o}(12 / 25 / 21)=3,121,000 ; \\
& t_{R}(12 / 25 / 21-1 / 31 / 22) \approx 7.636 \text { days } \\
& t_{d b l}(12 / 25 / 21-1 / 31 / 22) \approx 5.293 \text { days } \\
& \alpha_{S}(12 / 25 / 21-1 / 31 / 22) \approx 0.03168 / \text { day } \\
& \delta_{o}(12 / 25 / 21-1 / 31 / 22) \approx 0.96 \times 10^{-3} / \text { day } .
\end{aligned}
$$

The $N_{o}(12 / 25 / 21)=3,121,000$ starting point for the behavior change in $N(t)$ is similar to the prior Summer 2021 Resurgence, which also showed a behavior change in $N(t)$ at $N_{o}(8 / 13 / 21)=3,200,000$. This commonality shows that the uninfected population altered their behavior at similar points, which is likely when some hospitals became overwhelmed. 
Figure 3 shows the total number of CoVID-19 cases, $N_{\text {tot }}(t)$, resulting from combining all waves of the CoVID-19 pandemic, from March 2020 through January 2022. Whenever the pandemic appeared to be beaten down, people became more complacent, allowing new CoVID-19 variants to spread, and the pandemic rose up again, almost with every season.

The daily number of new cases $d N_{\text {tot }}(t) / d t$ in Figure 3 has peaks for the initial Spring 2020 pandemic; a Summer 2020 resurgence; the long Winter 2020 Resurgence; a small uptick in Spring 2021; the Summer 2021 Resurgence; and now the Winter 2021 Resurgence.

Figure 4 presents a tabulated summary of all the model parameters that were derived for each CoVID-19 wave. It shows these overall features:

(a) The $t_{d b l}=t_{R}(\ln 2)$ doubling times for several CoVID-19 waves vary only from $\{5.3-5.6$ days $\}$.

(b) The $\alpha_{S}$ Social Distancing and vaccination parameter for this CoVID-19 wave is one of the largest values, except for the early 2020 pandemic start.

(c) Given a fast-rising CoVID-19 pandemic Resurgence, the uninfected population generally takes notice of the severity of a new CoVID-19 wave when the new infections reach a level of $\sim 3,000,000$ above its baseline.

(d) The mask-wearing parameter, $\delta_{o}$, models the width and duration of the post-peak tail. Increased mask-wearing remains one the most powerful factors for hastening the CoVID-19 pandemic end.

\section{Summary}

Since our CoVID-19 modeling has been successful at predicting the time evolution of each USA CoVID-19 wave $^{\mathbf{1 - 7}}$, using the same few parameters:

$$
N(t) \approx \max \left[N_{o} \exp \left\{+\left(t /\left[t_{R}\left(1+\alpha_{S} t\right)\right]\right) \exp \left(-\delta_{o} t\right)\right\}\right],
$$

it indicates that the response of the $U(t)$ uninfected population was similar for each wave, even if different dominating factors drive each new resurgence.

The initial stage of the USA Winter 2021 CoVID-19 Resurgence started with the Omicron CoVID-19 variant becoming dominant. Our prior medrxiv.org preprint $^{7}$, covering data from $11 / 15 / 21-12 / 30 / 21$, showed that this initial stage was characterized by:

$$
\begin{aligned}
& N_{o}(11 / 15 / 21)=107,000 ; \\
& t_{R}(11 / 15 / 21-12 / 30 / 21)=8.05 \text { days } \\
& \alpha_{S}(11 / 15 / 21-12 / 30 / 21)=0.011 / \text { days } \\
& N(t=12 / 25 / 21) \approx 3,372,300 .
\end{aligned}
$$

These $\left\{N_{o} ; t_{R} ; \alpha_{S}\right\}_{11 / 15 / 21}$ model parameters are very similar to the prior USA Winter 2020 CoVID-19 Resurgence, when the USA only had a fullyvaccinated rate of $\sim 0.3 \%$. Since the USA now has a significantly vaccinated population, this new Winter 2021 Resurgence Omicron CoVID-19 variant is likely evading the vaccines. Since the $N_{o}(12 / 25 / 21)$ starting point of Eq. [2.1a] was nearly six weeks after the Eq. [3.2a] starting point, those two values should be similar, as they are. 
Just after the Christmas Holiday, the uninfected population responded to this CoVID-19 surge with enhanced Social Distancing and mask-wearing. The 12/25/21-1/31/22 post-Christmas data gives Eq. [2.1a]-[2.1e] as model parameters for this latter portion of the USA Winter 2021 CoVID-19 Resurgence, along with these updated $N(t)$ predictions for this CoVID-19 wave by itself:

$$
\begin{array}{lll}
N[t=1 / 31 / 22] & \approx 26,870,400 ; & {[3.4 \mathrm{a}]} \\
N[t=3 / 21 / 22] \approx 50,522,800 ; & {[3.4 \mathrm{~b}]} \\
N[t=6 / 10 / 22] \approx 60,329,500 ; & {[3.4 \mathrm{~b}]}
\end{array}
$$

with this wave no longer infecting practically everyone. Combining these results with the prior medrxiv.org preprint ${ }^{7}$ data, gives these updated USA CoVID-19 projections for the total number of USA CoVID-19 cases:

$$
\begin{array}{lll}
N_{\text {tot }}(t=[1 / 31 / 22]) \approx 77,122,000 \text { vs } N_{\text {data }}=75,399,650 ; & & {[3.5 \mathrm{a}]} \\
N_{\text {tot }}(t=[3 / 21 / 22]) \approx 101,476,500 ; & {[3.5 \mathrm{~b}]} \\
N_{\text {tot }}(t=[6 / 10 / 22]) \approx 111,531,800 ; & {[3.5 \mathrm{c}]}
\end{array}
$$

from all USA CoVID-19 waves, assuming no new CoVID-19 resurgence intervenes. More mask-wearing would further mitigate CoVID-19 spread, and it could significantly reduce these projected future USA CoVID-19 totals.

\section{List of Figures}

Fig. 1: The Initial USA CoVID-19 Winter 2021 Resurgence, by Itself

Fig. 2: USA CoVID-19 Winter 2021 Resurgence Post-Christmas Update

Fig. 3: USA CoVID-19 Totals: 3/21/2020 through 1/31/2022

Fig. 4: Summary of CoVID-19 Model and Parameter Values

\section{$5 \quad$ References}

1. https://medrxiv.org/cgi/content/short/2020.05.04.20091207v1

"Initial Model for the Impact of Social Distancing on CoVID-19 Spread"

2. https: //medrxiv.org/cgi/content/short/2020.06.30.20143149v1

"Orthogonal Functions for Evaluating

Social Distancing Impact on CoVID-19 Spread"

3. https: //medrxiv.org/cgi/content/short/2020.08.07.20169904

"Model to Describe Fast Shutoff of CoVID-19 Pandemic Spread"

4. https://medrxiv.org/cgi/content/short/2020.09.16.20196063

"Initial Model for USA CoVID-19 Resurgence"

5. https: //medrxiv.org/cgi/content/short/2021.08.16.21262150

"The IHME vs Me: Modeling USA CoVID-19 Spread, Early Data to the Fifth Wave"

6. https: //medrxiv.org/cgi/content/short/2021.10.15.21265078

"Updated Model for the USA Summer 2021 CoVID-19 Resurgence"

7. https: //medrxiv.org/cgi/content/short/2022.01.06.22268868

"Modeling the USA Winter 2021 CoVID-19 Resurgence"

8. www.bing.com/covid/local/unitedstates?form $=$ COVD07 


\section{Fig. 1: The Initial USA CoVID-19 Winter 2021 Resurgence, by Itself}

\section{Initial USA Winter 2021 Omicron CoVID-19 Wave By Itself}

First Deviation from Prior Models: 11/15/2021

\section{$1.0 \mathrm{E}+08$}

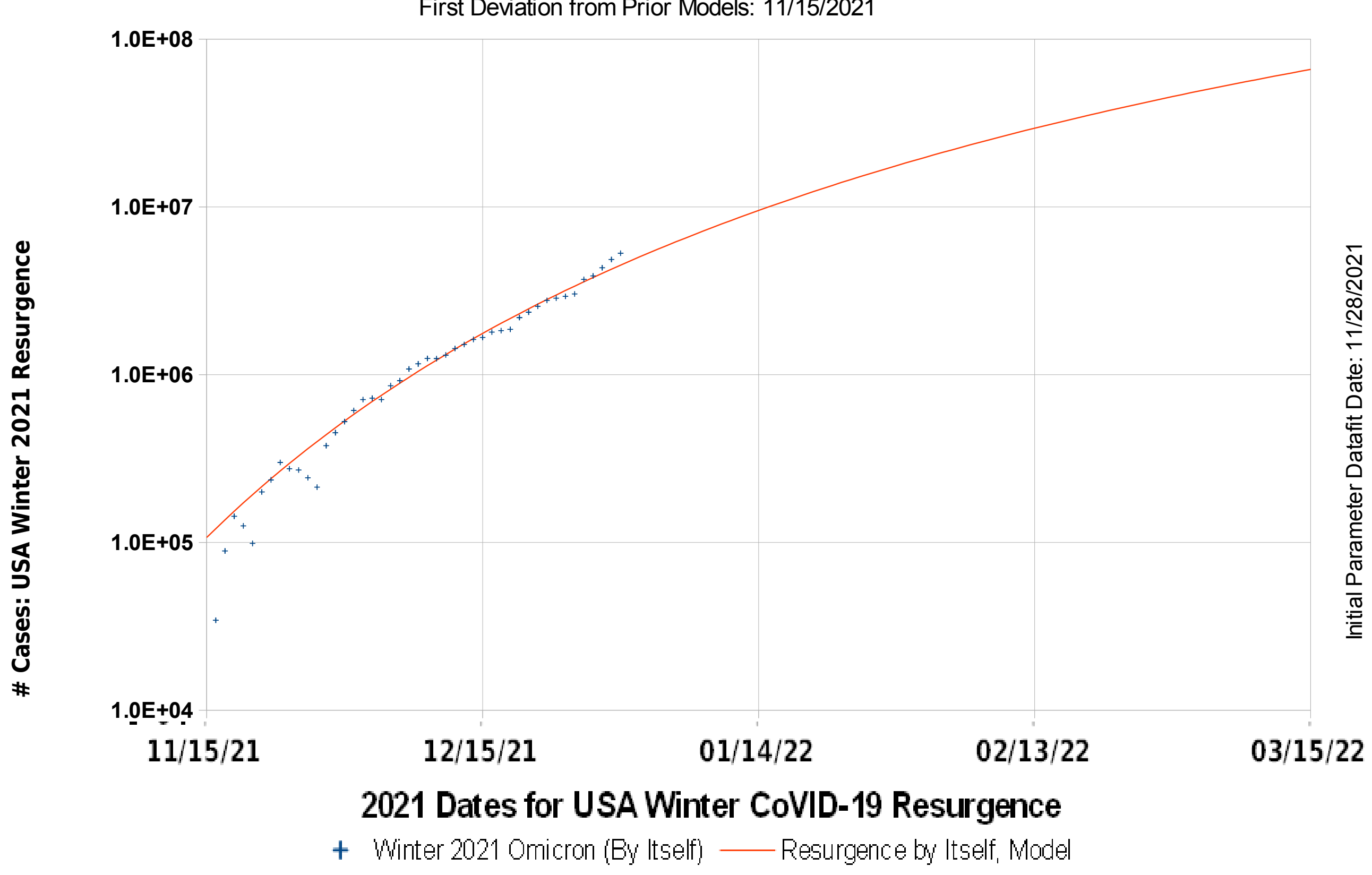

D


Fig. 2: USA CoVID-19 Winter 2021 Resurgence Post-Christmas Update

\section{USA Winter 2021 Omicron CoVID-19 Wave By Itself}

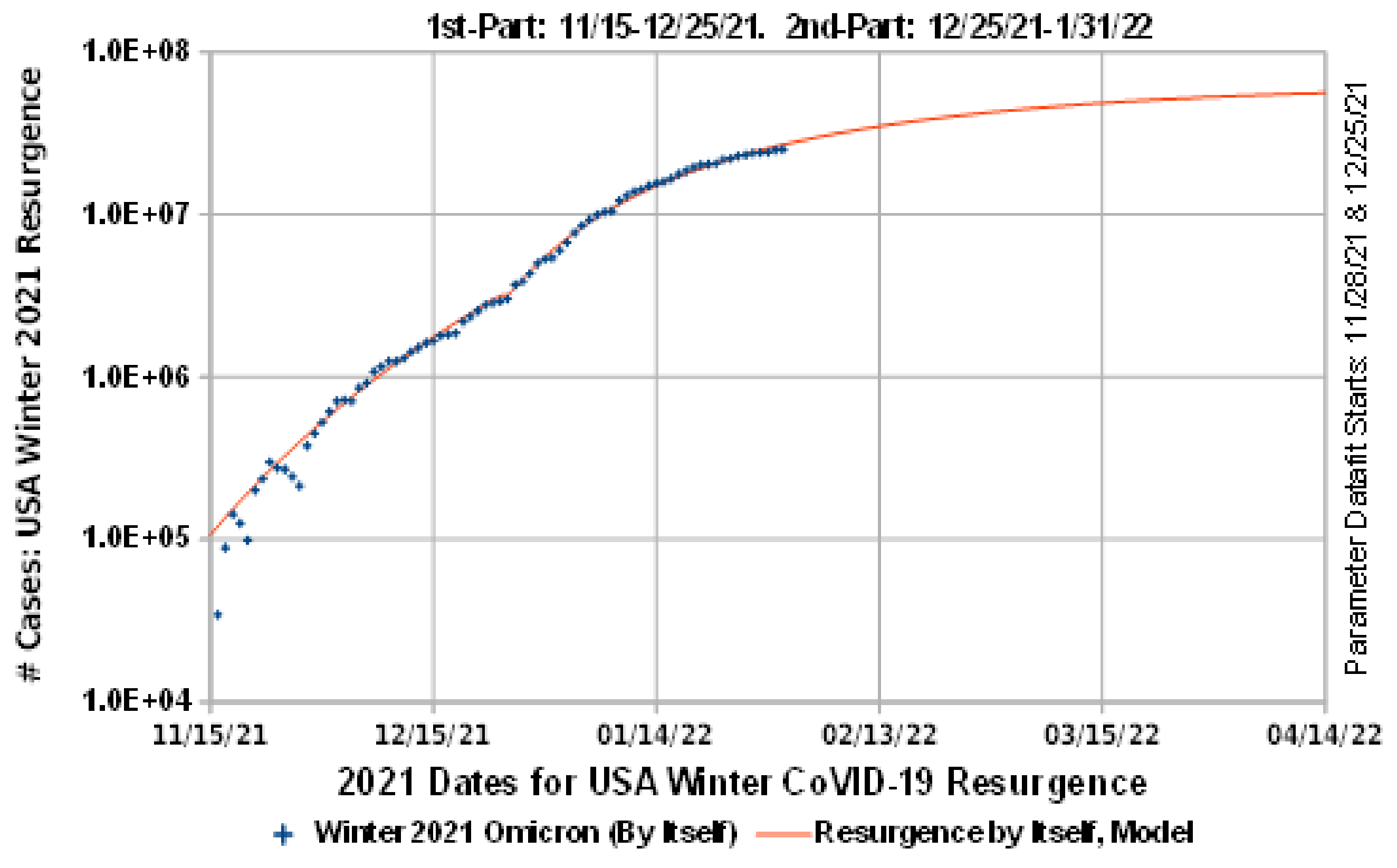




\section{Fig. 3: USA CoVID-19 Totals: 3/21/2020 through 1/31/2022}

USA CoVID -19 Data vs Models, 3/21/2020 through 1/31/2022

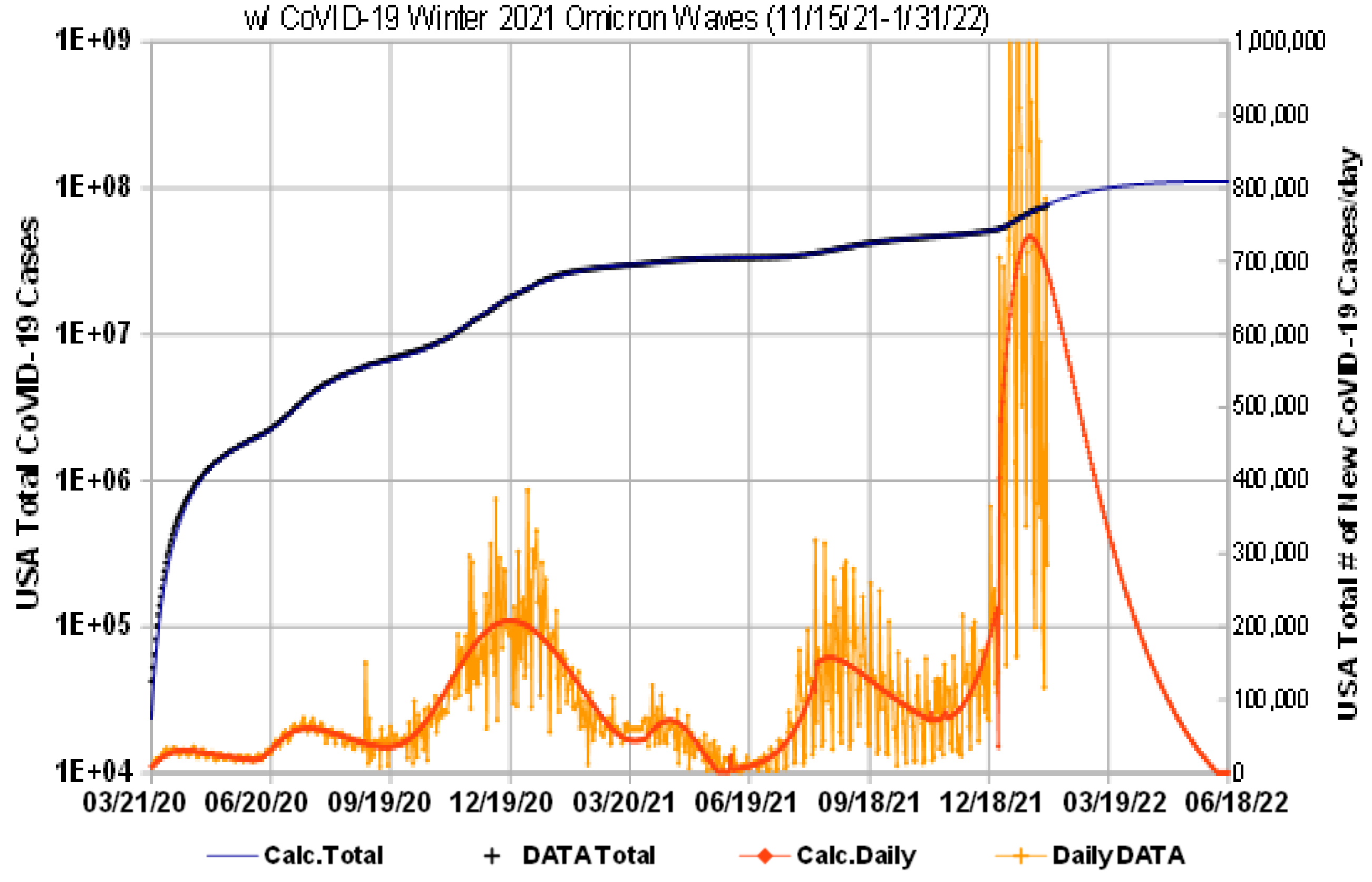




\section{Fig. 4: Summary of CoVID-19 Model and Parameter Values}

\begin{tabular}{|c|c|c|c|c|c|c|c|}
\hline Index & $\begin{array}{c}\text { U.S.A. CoVID } \\
19 \text { Stage }\end{array}$ & $\begin{array}{l}\text { Mo, (Initial } \\
=0 \text { Value) }\end{array}$ & $\begin{array}{c}t=0 \\
\text { Date }\end{array}$ & $\begin{array}{c}t / d b \| \\
d a y s \text { at } \\
t=0\end{array}$ & $\underbrace{}_{R}$ & $\begin{array}{c}\alpha_{S} \\
\text { value }\end{array}$ & $\begin{array}{c}\delta_{0} \\
\text { value }\end{array}$ \\
\hline 1 & $\begin{array}{l}\text { Ini. Model } 67 / 20 \\
\text { Upuate }\end{array}$ & 23,710 & 0321,2020 & 1.99657 & 2,88044 & 0.06618 & 0.00000 \\
\hline 2 & $\begin{array}{l}1^{\text {th }} \text { Resurgence } \\
\text { Summer } 2020 \Rightarrow\end{array}$ & 15,650 & 06072020 & 2.80000 & 4.15496 & 0.058 & 0.0108 \\
\hline 3 & $\begin{array}{c}\text { Win ter } 2020 \\
\text { Riesurgence } \Rightarrow\end{array}$ & 88,900 & $09,25,2020$ & 5.57983 & 8.05 & 0.011 & 0.001748 \\
\hline 4 & $\begin{array}{l}\text { Small spring } 2021 \\
\text { Riesurgence } \Rightarrow\end{array}$ & 146,000 & $03 / 192021$ & 5.57983 & 8.05 & 0.00128 & 0.01365 \\
\hline 5 & $\begin{array}{l}\text { Inital Portion, } \\
\text { Summer } 2021 \\
\text { Riesurgence } \Rightarrow\end{array}$ & 41,000 & $06,07,2021$ & 9.08924 & 13.113 & 0.0030 & 0.00000 \\
\hline 6 & $\begin{array}{c}\text { Latter Portion, } \\
\text { Summer } 2021 \\
\text { Resurgence } \Rightarrow\end{array}$ & $3,200,000$ & $08 / 132021$ & 14.4339 & 20.83740 & 0.019999 & 0.000489 \\
\hline 7 & $\begin{array}{l}\text { Initial Fortion, } \\
\text { Wirter } 2021 \\
\text { Resurgence } \Rightarrow\end{array}$ & 107,000 & $11,45,2021$ & 5.57983 & 8.05 & 0.011 & 0.000001 \\
\hline 8 & $\begin{array}{l}\text { Latter Fortion, } \\
\text { 'Winter } 2021 \\
\text { Resurgence }\end{array}$ & $3,121,000$ & $12,25,2021$ & 5.29287 & 7.636 & 0.03168 & 0.00096 \\
\hline
\end{tabular}

